\author{
Military Technical College \\ Cairo, Egypt
}

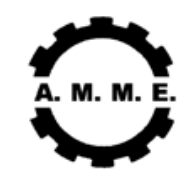
$12^{\text {th }}$ International Conference on Applied Mechanics and Mechanical Engineering (AMME)

\title{
DYNAMIC IMPACT PROBLEMS: MODELING AND ANALYSIS
}

\author{
Elhady* A.M.
}

\begin{abstract}
Solid mechanics investigators gave the dynamics of bodies in impact special interest long time ago. Many approaches were developed to solve this problem since $F$. Neumann (1885). Reviewing these various approaches revealed that the impact body usually regarded as a system possessing one degree of freedom and the aim is to seek the configuration of maximum compression when the body starts to achieve instantaneous equilibrium. This paper is aimed to model the impacted body as a system possessing multi degrees of freedom by using the finite element method. The normal and oblique impacts are discussed taking into consideration the neighborhood area of the instantaneous impact point is smooth or rough. The effect of the number of elastic modes of vibration on the impact response is considered.
\end{abstract}

\footnotetext{
* Department of Space Science, National Authority of Remote Sensing and space Science, Cairo, Egypt.
} 


\section{INTRODUCTION}

Dynamics of mechanical systems are commonly used models of rigid body. However they have number of limitations and difficulties that are not usually appreciated when applied to problems involving impact and Coulomb friction. The presence of these difficulties is imposed due to the fact that no body can be considered perfectly rigid where all bodies are deformed to some extent when they go beyond contact with an obstacle. The classical approximation of longitudinal impact of elastic rod against a rigid obstacle was described by Newmann (1885) in terms of a system of $2^{\text {nd }}$ order partial differential equations. These equations were reduced to a single D'Alembert equation. Solution of this equation is not simple, especially when impact frequently occurs, the rod cross-section is variable, or produced stresses exceed the elastic limit. Since that time many authors attempt to describe this problem by different approaches [1-5]. Contact with a rigid obstacle is widely common approximate by contact with spring-like body in studying elastic bodies carried out by Kikuchi and Oden in1988 [6]. Spring-like approximations are used to obtain results for certain kinds of contact models of elastic bodies, where springs do not have inertia and each point of contact have only one degree of freedom. On the other hand elastic bodies do have inertia and their description is infinite dimension.

In the last two decades mathematicians and engineers gave this problem special interest

Stewart [7-10] presented an approach to model bodies in impact come out of work on rigid body dynamics. Solberg \& Papadopoulos [11] formulate the problem in a psedorigid body model. Schatzman [12] approximated the dynamical impact problem in generalized coordinates by penalty method, which is often used for numerical approximation. His approximation includes smooth time-dependent set of constraints and the possibility of zero restitution coefficients. Glocker [13] established the contact kinematical equations and the impact equations. furthermore, Newton's and Poisson's impact laws were studied in inequality form for one collision point and extended by superposition to multi-contact configurations. Paoli [14] considered vibro-impact problems of mechanical systems with finite number of degrees of freedom submitted to perfect unilateral constrains. The model is basically described by a second-order measure differential inclusion for the unknown position completed with a constitutive impact law. Mahmoud et al. [15] developed an incremental finite element model to simulate the dynamic behavior of elastic bodies in contact. They solve the direct impact of similar and dissimilar rods. There model solution based on finite element simulation. Moreover, Hughes et. al. [16] solved the problem of classical impact contact problem based on finite element.

\section{CONTACT IMPACT MODELS}

There are at least two historical approaches to impacts: Newton's kinematics impact law, which reverses the sign of the relative velocity at the impact and takes into account dissipation by a coefficient $0 \leq \varepsilon \leq 1$

$$
\varepsilon(v-V)=(C-C)
$$


Newton's impact law is indeed not an independent equation, but it can be derived from the conservation of linear momentum of the actually one-dimensional system of two point masses under the hypothesis of the law of interaction and the assumption of a dissipative contact process. Newton's impact law is derived and validated independent of the contact duration and of any temporal distribution of the contact forces.

Poisson's impact law, which requires a decomposition of the impact process into a compression phase (-) and a decompression phase $(+)$ in order to define the restitution coefficient $\varepsilon$ by the ratio of the corresponding impulsive forces,

$$
\Lambda^{+}=\varepsilon \Lambda^{-}
$$

Poisson impact law is based on Newton's second law and Euler's axiom regarding the 'normal force' at the impact. This is already close to the common applied methods of treating impacts, where the impact law is handled as an independent equation, which can generally be derived from any mechanical principle when dealing with rigid bodies.

\subsection{Impact kinematics}

This section will concern by a brief study to the kinematics of contacting bodies as far as it is needed for impact. This requires in particular the definitions of the contact points and the normal relative velocity to set up unilateral constraint and to formulate an impact law.

We consider a non-moving strictly convex rigid body and choose a body-fixed point $\mathrm{P}$ as the point of reference. The surface $\Sigma$ of the body is assumed to be smooth and given in a regular parametric form with displacements $\rho\left(\xi^{1}, \xi^{2}\right)$ relative to $P$ (Fig 1a). The trihedral $\left(t_{1}, t_{2}, n\right)$ at point $\rho\left(\xi^{1}, \xi^{2}\right)$ of the surface is obtained via
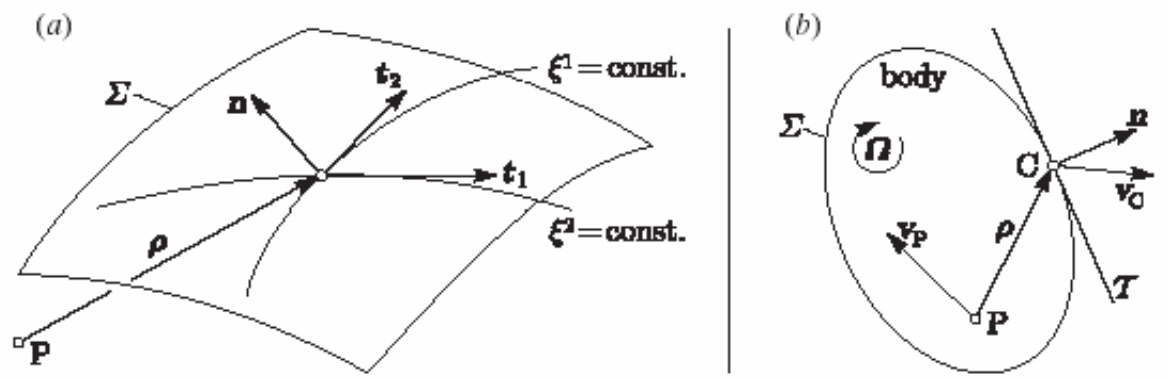

Fig. 1. Surface parameterization and rigid-body kinematics

$$
t_{1}=\frac{\partial \rho}{\partial \xi^{1}} \quad t_{2}=\frac{\partial \rho}{\partial \xi^{2}} \quad n=\frac{t_{1} \times t_{2}}{\left|t_{1} \times t_{2}\right|}
$$

where $\left(\xi^{1}, \xi^{2}\right)$ are arranged such that the unit vector $\mathrm{n}$ becomes the outward normal. Assume now that the body is moving with the linear velocity $v_{p}(t)$ of the point of reference $P$ and the angular velocity $\Omega(t)$ (Fig $1 b)$. Furthermore, let $\xi^{\alpha}(\alpha=1,2)$ depend on time $t$, which makes $\rho$ and $n$ also change relative to the body. By Euler's formula for rigid-body kinematics we obtain the absolute changes in time $\rho, n$ of $\rho, \mathrm{n}$ as follow: 


$$
\begin{aligned}
& \dot{\rho}-\Omega \times \rho=\frac{\partial \rho}{\partial \xi^{\alpha}} \dot{\xi}^{\alpha} \\
& \dot{n}-\Omega \times \rho=\frac{\partial n}{\partial \xi^{\alpha}} \dot{\xi}^{\alpha}
\end{aligned}
$$

Denote $v_{C}$ the velocity of the body-fixed point $C$ with displacement $\rho$ relative to $P$. Employing the rigid-body velocity formula,

$$
\mathrm{v}_{\mathrm{c}}-\mathrm{v}_{\mathrm{p}}=\Omega \times \rho
$$

we can rewrite the absolute time change of $\rho$ as follow:

$$
\dot{\rho}=v_{c}-v_{p}+\frac{\partial \rho}{\partial \xi^{\alpha}} \dot{\xi}^{\alpha}
$$

Noting that $\rho$ differs from $\left(\mathrm{v}_{\mathrm{c}}-\mathrm{v}_{\mathrm{p}}\right)$ only in the tangential terms.

Now, the main equations of contact kinematics collisions are able to be derived which present the definition of the contact points including their gap function and their relative velocity. Therefore, two rigid bodies are considered and referenced by right lower indices 1 and 2 as depicted in Fig. 2, performing the following construction.

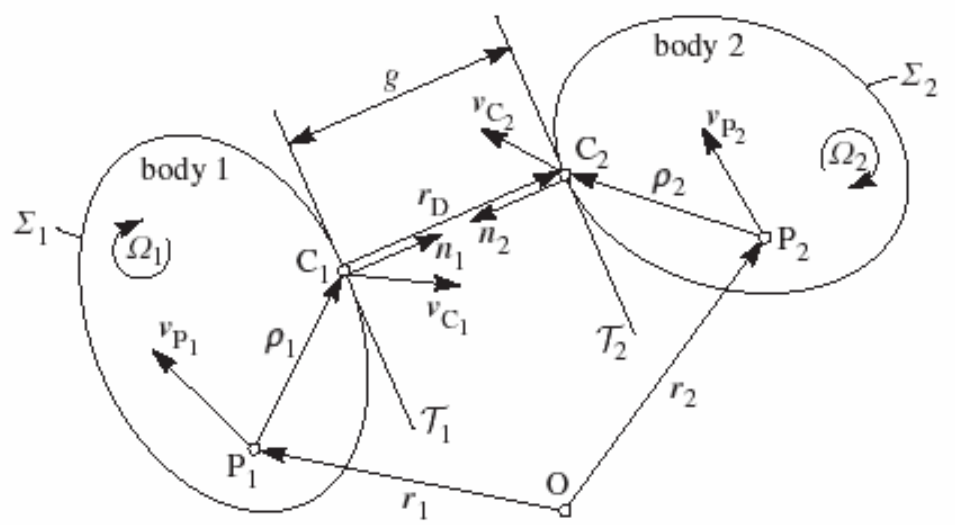

Fig.2. Kinematics Parameters of Two Bodies in Impact

A distance vector $r_{D}$ can be introduced as:

$$
r_{D}=\left(r_{2}+\rho_{2}\right)-\left(r_{1}+\rho_{1}\right)
$$

The two tangential planes $\tau_{\mathrm{i}}$ are constructed in a parallel manner so that $\mathrm{r}_{\mathrm{D}}$ becomes collinear with each of the normal $n_{i}$.

The gap function $g=n_{i}^{T} r_{D}$ is defined by the minimum distance between the contacting points which are $\rho_{i}\left(\bar{\xi}_{i}^{\alpha}\right)$. The contact points are defined as the four surface parameters $\bar{\xi}_{i}^{\alpha}$ which are the solutions of the four nonlinear equations

$$
\left.\begin{array}{l}
n_{1}^{T} t_{\alpha 2}=0 \\
t_{\alpha 1}^{T} r_{D}=0
\end{array}\right\} \quad \alpha=1,2
$$


The gap function of the contact points can be defined with the following properties. When the bodies are properly separated the gap function should be $g>0$ with a magnetude equal to the minimum value of all distances between any point of body 1 and any point of body 2 . For contacting bodies the gap function should be $g=0$. Hence, $g \geq$ 0 defines a unilateral constraint. Now, differentiation of $g$ with respect to time leads to the normal relative velocity i.e. the velocity on which the classical impact laws are based.

$$
\dot{g}=n_{1}^{T} \dot{r}_{D}+\dot{n}_{1}^{T} r_{D}
$$

Gap time change rate may further be simplified particularly when $\dot{n}_{1}^{T} r_{D}=0$ by construction. The remaining term becomes

$$
\begin{aligned}
n_{1}^{T} \dot{r} & =n_{1}^{T}\left(\left(\dot{r}_{2}+\dot{\rho}_{2}\right)-\left(\dot{r}_{1}+\dot{\rho}_{1}\right)\right) \\
& =n_{1}^{T}\left(\left(v_{c 2}+\left(t_{\alpha} \dot{\xi}^{\alpha}\right)_{2}\right)-\left(v_{c 1}+\left(t_{\alpha} \dot{\xi}^{\alpha}\right)_{1}\right)\right. \\
& =n_{1}^{T}\left(v_{c 2}-v_{c 1}\right)
\end{aligned}
$$

where $v_{c i}$ are the absolute velocities of the rigid-body contour points $C_{i}$ placed at $\rho_{i}\left(\bar{\xi}_{i}^{\alpha}\right)$ at instant time t.

\subsection{Impact kinetics}

After having established the equations of contact cinematically the impact equations of the system is now planning to be stated. Consider two bodies with already given contact points, oriented normals $\boldsymbol{n}_{\mathrm{i}}$ and tangent planes $\boldsymbol{T}_{\mathrm{i}}$ as depicted in Fig. 3. Starting investigations with the impact-free motion followed by developing the contact model and the contact forces in the classical Newton-Euler equations are taken into account. These equations are then reformulated as equalities of measures in order to allow atomic quantities to express velocity jumps and impulsive forces when integration with respect to time is performed. The resulting equation balances at each single point in time the instantaneous changes in the generalized momentum and the impulsive generalized forces of the system if any, and is called the impact equation.

Our contact model is based on the assumption that the force interaction of the two bodies at the contact points is frictionless. The contact forces $F_{\mathrm{C} i}$ are therefore collinear with the normals $\boldsymbol{n}_{\mathrm{i}}$ and, by the principle of interaction, of the same size but of opposite direction, $\boldsymbol{F}_{\mathrm{C} 1}=-\boldsymbol{F}_{\mathrm{C} 2}$. Therefore, $\boldsymbol{F}_{\mathrm{C} i}$ can be expressed as

$$
F_{C 1}=-n_{1} \lambda, \quad F_{C 2}=n_{1} \lambda,
$$

where $\boldsymbol{n}_{\mathrm{i}}$ are normalized vectors. The multiplier $\lambda$ is the scalar value of the contact forces which acts as compressive forces when $\lambda>0$ and reflects tension for $\lambda<0$.

Assume now a system of $\mathrm{n}$ rigid bodies with one contact between body 1 and body 2 . The virtual work $\delta \mathrm{W}$ of the system to obtain the projected Newton-Euler equations including the virtual work of the contact forces, 


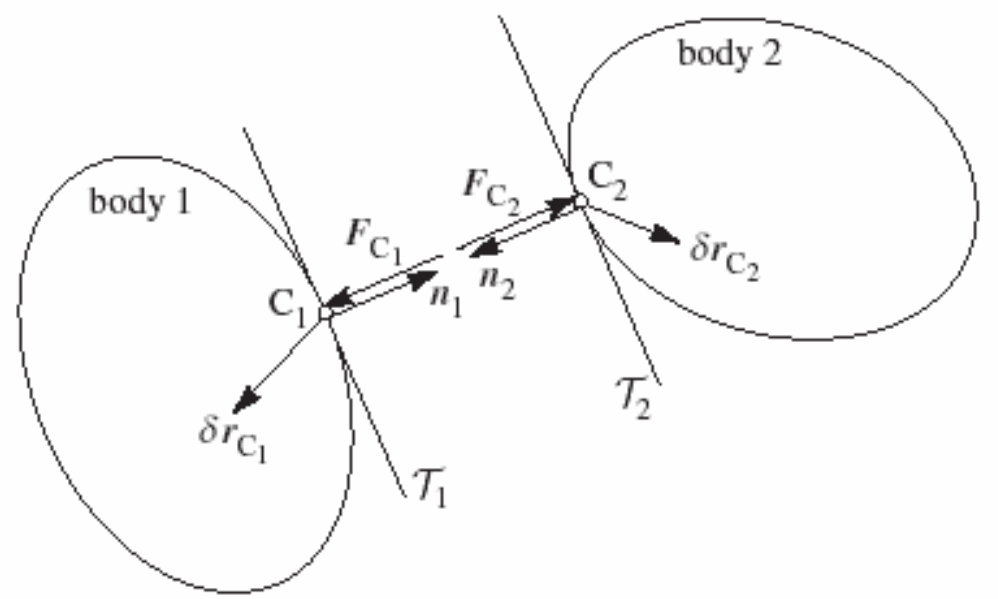

Fig.3. Kinetics parameters of two bodies in impact

$$
\begin{array}{r}
\delta W=\sum_{i=1}^{n}\left[\delta r_{S}^{T}\left(m a_{S}-F\right)+\delta \Phi^{T}\left(\Theta_{S} \Psi+\Omega \times \Theta_{S} \Omega-M_{S}\right]_{i}+\delta r_{C_{1}}^{T} F_{C_{1}}+\delta r_{C_{2}}^{T} F_{C_{2}}=0\right. \\
\forall \text { admissible } \delta r_{(\cdot)}, \delta \Phi .
\end{array}
$$

In this expression one discover for each body Newton's second law

$$
m a_{s}-F=0
$$

And Euler's axiom

$$
\Theta_{S} \Psi+\Omega \times \Theta_{S} \Omega-M_{S}=0
$$

where

$m \quad$ is the mass of the corresponding body,

$a_{S} \quad$ is the acceleration of the center of mass $S$,

$F \quad$ is the of the bodies resultant external forces,

$\Theta_{S} \quad$ is the inertia operator with respect to point $S$,

$\Psi \quad$ is the angular acceleration, $\Omega$ the angular velocity and

$M_{S} \quad$ is the resultant of the bodies external free and induced moments with respect to point S. $\delta r_{S}$ is the virtual translation of the center of mass $S$ and

$\delta \Phi \quad$ is the virtual rotation of the body.

$\delta r_{C_{i}}^{T} F_{C_{i}}$ is the virtual work due to translation of the contact force acts at the body contour point $\mathrm{C}_{i}$.

Let $J_{C_{i}}$ are the Jacobians of the body contour points $C_{i}, J_{R}$ and $J_{S}$ denote the Jacobians of rotation and translation of point $S$ of the investigated body, and $\bar{i}_{S}, \bar{i}_{R}$ are obtained from differentiation of the corresponding velocities and depend on $q, \dot{q}, t$. Now, the virtual work of the system can be written as follows:

$\delta W=\delta q^{T} \sum_{i=1}^{n}\left[J_{S}^{T} m J_{S} \ddot{q}-J_{S}^{T}(\ldots)+J_{R}^{T} \Theta_{S} J_{R} \ddot{q}+J_{R}^{T}(\ldots)\right]_{i}+\delta q^{T}\left(J_{C 2}-J_{C 1}\right)^{T} n_{1} \lambda=0 \quad \forall \delta q$.

This leads to the projected Newton-Euler equations 
$M(q, t) \ddot{q}-h(q, \dot{q}, t)-w(q, t)=0 ;$

where the mass matrix

$$
M=\sum_{i=1}^{n}\left[J_{S}^{T} m J_{S}+J_{R}^{T} \Theta_{S} J_{R}\right]_{i}
$$

the gyroscopically acceleration vector

$$
-h=\sum_{i=1}^{n}\left[J_{S}^{T}(\ldots)+J_{R}^{T}(\ldots)\right]_{i} ; \text { and }
$$

the generalized force direction as already introduced.

$$
w=\left(J_{C_{2}}-J_{C_{1}}\right)^{T} n_{1}
$$

Note that the projected Newton-Euler equations require the existence of velocities $q$ and accelerations $\ddot{q}$, both being meaningless for the event of impact. One has therefore to replace Newton-Euler equations by a more suitable formulation, which also takes into account the impacts as follows:

$$
M(q, t) d u-h(q, \dot{q}, t) d t-w(q, t) d \Lambda=0
$$

Here the velocities denoted by $u(t)$ are assumed to be functions of bounded variations. The displacement $q(t)$ are obtained from integration of $u(t)$. The probably 'infinite values'

of the acceleration $\ddot{q}$ and the force $\lambda$ at the impact are taken into account by the deferential measure $d u$ of $u$ and by the force measure $d \Lambda$. In order to now obtain the impact equations we integrate Newton-Euler over singleton $\left\{\mathrm{t}_{\mathrm{o}}\right\}$ and denote $\mathrm{q}_{\mathrm{o}}=\mathrm{q}\left(\mathrm{t}_{\mathrm{o}}\right)$, $\int_{t_{0}} d u=u^{+}\left(t_{0}\right)-u^{-}\left(t_{0}\right)$, and $\int_{t_{0}} d \Lambda=\Lambda\left(t_{0}\right)$ defines the value of the impulsive force at $t_{0}$. This yields:

$$
\begin{aligned}
& \int_{t_{0}}(M(q, t) d u-h(q, u, t) d t-w(q, t) d \Lambda=0 \\
& M(q, t)\left(u^{+}\left(t_{0}\right)-u^{-}\left(t_{0}\right)\right)-w\left(q_{0}, t_{0}\right) \Lambda\left(t_{0}\right)=0
\end{aligned}
$$

The resulting expression is called the impact equation of the system.

Poisson's impact law requires a decomposition of the impact process into two succeeding phases. The first is the compression phase and the second is the decompression phase. Compression starts with the pre-impact velocity denoted by (upper index -). Decompression events with the velocity stats at the end of compression and terminates with the post-impact velocities denoted by (upper index + ). This yields the equation of the compression phase,

$M\left(u^{0}-u^{-}\right)=w \Lambda^{-}$,

and for the decompression phase,

$M\left(u^{+}-u^{0}\right)=w \Lambda^{+}$

where $u^{0}$ is post-compression velocity. The impulse at decompression phase $\Lambda^{+}$can be determined by Poison's law as a function of the impulse at compression phase $\Lambda^{+}=\varepsilon \Lambda^{-}$. 


\section{PROBLEM FORMULATION}

\subsection{General formulation}

To describe the configuration of a mechanical system we use a vector $\mathbf{q}$ of generalized coordinates; these can include things like the position of the center of mass in $\mathbb{R}^{3}$, orientations represented as orthogonal matrices $Q \in \mathbb{R}^{3 \times 3}$ or unit quaternion, angles of hinges or other joints, amplitudes of elastic modes, or deformation matrices for pseudorigid bodies .. etc. If we have two mechanical subsystems each with their own generalized coordinates $\mathbf{q}_{1}$ and $\mathbf{q}_{2}$, then the configuration of the combined system is described by the vector $\mathbf{q}=\left[\mathbf{q}_{1}^{\top}, \mathbf{q}_{2}^{\top}\right]^{\top}$. For hinges and joints there may be some bilateral (equality) constraints: $\mathbf{g}(\mathbf{q})=0$. Note that $\mathbf{g}$ may be a vector-valued function. However, we expect that the dimension of $\mathbf{g}(\mathbf{q})$ is less than the dimension of $\mathbf{q}$.

For contact problems we are interested in unilateral (inequality) constraints $h(q) \geq 0$. For a single possible contact we need only a scalar function $\mathrm{h}$, but for more complex systems we will need a vector function $h(q)$. In that case, $h(q) \geq 0$ is interpreted as meaning $h_{i}(q) \geq 0$ for all $i$. These functions define the admissible contact region $C$ :

$$
\mathrm{C}=\{\mathrm{q} \mid \boldsymbol{g}(\mathrm{q})=0, \mathrm{~h}(\mathrm{q}) \geq 0\} \text {. }
$$

Contact occurs when $h_{i}(q)=0$ for some $i$. If $h_{j}(q)>0$ for all $i$, then we can locally ignore the inequality constraints. There is a normal contact force which prevents $q$ leaving $C$ is a linear combination of the vectors $\nabla g_{j}(q)$ and $\nabla h_{j}(q)$. Since the normal contact force must point inward into $C$, the coefficients of $\nabla h_{i}(q)$ have to be non-negative.

In keeping with the use of generalized coordinates, we use a Lagrangian formulation of mechanics: the Lagrangian function is

$$
L(q, v)=\frac{1}{2} v^{T} M(q) v-V(q) .
$$

The Euler-Lagrange equations of motion are therefore

$$
\frac{d}{d t} \frac{\partial L}{\partial v}-\frac{\partial L}{\partial q}=f_{\text {ext }}+f_{\text {contact }} \text {, }
$$

where the $f_{\text {ext }}$ are the external forces and $f_{\text {contact }}$ are the contact forces.

The contact force can be separated into normal and frictional tangential forces, and parameterize the friction component

$$
f_{\text {contact }}=\sum_{j} n^{j}(q) C_{n}^{j}+D^{j}(q) \beta^{j}
$$

where the summation is overall potential contact:

$n^{j}(q)$ is the outward normal vector at $\mathrm{j}^{\text {th }}$ contact point,

$D^{j}(q)$ is the matrix of direction vectors,

$C_{n}^{j} \quad$ is the normal contact force at $\mathrm{j}^{\text {th }}$ contact point, and

$\beta^{j} \quad$ is the friction force at $\mathrm{j}^{\text {th }}$ contact point.

The maximum dissipation principle [6] sates that we chose $\beta^{j}$ at time $t$ to maximize

$$
-\left(\beta^{j}\right)^{T} D^{j}(q(t))^{T} v\left(t^{+}\right)
$$


subject to the condition:

$$
\beta^{j} \in C_{n}^{j} F C_{j}^{0}(q)
$$

where

$F C_{j}^{0}(q) \quad$ is the possible friction force for unit normal contact force.

This is a linear minimization problem with convex constrains.

Combining Euler-Lagrange Equations of motion, with Kuhn-Tucker conditions, the main contact condition and Newtonian impact law for single contact give the following impact model:

$$
L(q, v)=\frac{1}{2} v^{T} M(q) v-V(q),
$$

Subject to

$$
\begin{aligned}
& 0 \leq C_{n}^{j} \perp g_{j}(q) \geq 0, \quad \text { for all } j, \\
& \left.0=\left(C_{n}^{j}\right)^{\gamma_{j}} D^{j}(q(t))^{T} v\left(t^{+}\right)\right)+\lambda_{j} \nabla \psi_{j}\left(\beta^{j}\right), \quad \text { for all } j, \\
& 0 \leq \lambda_{j} \perp\left(C_{n}^{j}\right)^{\gamma_{j}}-\psi_{j}\left(\beta^{j}\right) \geq 0, \quad \text { for all } j \\
& n^{j}(q(t))^{T} v\left(t^{+}\right)=-\varepsilon^{j} n^{j}(q(t))^{T} v\left(t^{-}\right) \text {if } g(q(t))=0 .
\end{aligned}
$$

where

$a \perp b$ means that $a^{\top} b=0$, or $a$ and $b$ are both scalars, $a b=0$.

$\psi_{j} \quad$ is non-negative homogeneous convex function which satisfies the property that there is $\gamma \geq 1$ such that for all $\beta$ and $\alpha \geq 0, \psi(\alpha \beta)=\alpha^{\gamma} \psi(\beta)$,

$\nabla \psi_{j}$ is the generalized gradient of the function $\psi_{j}$,

$\varepsilon \quad$ is the coefficient of restitution for the $\mathrm{j}^{\text {th }}$ contact and can have values from zero to one inclusive,

$g_{j}(q)$ is the gap for the $j^{\text {th }}$ contact,

$k(q, v)$ is the elastic strain energy.

$\frac{d q}{d t}=v$

\subsection{Convex programming formulation}

Impact response analysis is to describe the dynamic behavior of objects after a collision occurs. In order to obtain a continuous solution of the impact mechanics problem concerned in this paper, variational methods of approximation can be used. They include Raleigh and Ritz, Galerkin, Petrov-Galerkin (Weighted-residuals), and the finite element method. All these methods except the last one are traditional variational approaches which have three main shortcomings: 1) difficulty in handling irregular domain: 2) difficulty in selecting coordinate functions; 3) dependence of coordinate functions on the geometry of the domain. These limitations can be overcome by the finite element method which is a piecewise polynomial approximation of weak forms of boundary- or initial-value problems over a domain partition of the solution to the system. Locally, the finite element method represents a function as a polynomial in much the same spirit as the classical Lagrange and Hermit interpolation methods. In this paper, the proposed impact response analysis algorithm contains two key components: the forward Lagrange multiplier method (convex programming) and the local finite element 
method. Convex programming has recently found widespread use in the efficient formulation and solution of a multitude of engineering problems. Advantages of convex programming include its polynomial-time convergence to globally optimal solution, availability of highly efficient and reliable solvers, and the scale of problems that can be handled.

The Lagrange dynamics and the finite element method are used to describe the generalized motion because of their simplicity and flexibility. On the basis of D'Alembert's principle and the principle of virtual displacement, the semi-discrete system governing equations that describe the equilibrium of a system (Eq. (30)) is approximated by:

$$
[M]\{\ddot{q}\}+[C]\{\dot{q}\}+[K]\{q\}=\{R\}
$$

Where;

$[\mathrm{M}]$ is the Mass matrix,

[C] is the Damping matrix,

$[\mathrm{K}]$ is the stiffness matrix,

$\{\ddot{q}\} \quad$ is the acceleration vector,

$\{\dot{q}\}$ is the velocity vector,

$\{q\}$ is the movement vector, and

$\{R\} \quad$ is the external load vector.

For arbitrary impact between deformable objects, Lagrange multipliers may be introduced into the equation of motion as follows:

$$
[M]\{\ddot{q}\}+[C]\{\dot{q}\}+[K]\{q\}+[G]^{T}\{\Gamma\}=\{R\}
$$

[G] Geometric constraint matrix to prevent any penetration between colliding bodies, $\{\Gamma\} \quad$ Lagrange multiplier vector which represents the surface contact forces.

Mathematically, equation (36) represents a set of linear differential equations of second order and, it will be solved by using standard procedures for differential equations [17]. However, the procedures for the solution of general differential equations could become computationally expensive if the matrices are large unless some special methods are used by taking advantage of the characteristics of the matrices $\mathrm{K}$, and $\mathrm{M}[18,19]$. The direct integration method proposed by Hughes et. al. [20-22] will be considered as one of the few effective methods for the solutions of dynamic analysis. Naming the term 'direct' means that prior to the numerical integration no transformation of the equations into a different form is conducted. The basic ingredients in the implicit-explicit procedure are 1) a given implicit integrator, 2) a predictor-corrector explicit scheme constructed to be "compatible" with the given implicit integrator, and 3) a synthesis of the implicit and explicit schemes by way of modified time-discrete equation of motion. For the aim of efficiency the Newmark family will be used assuming positive $\eta$ which gives rise to implicit method. The proposed method is used as follows:

i- Corrector

$$
q^{n+1}=\tilde{q}_{n+1}+\theta \Delta t^{2} \ddot{q}_{n+1}
$$


ii- Predictor

$$
\dot{q}^{n+1}=\tilde{\dot{q}}_{n+1}+\eta \Delta t \ddot{q}_{n+1}
$$

$$
\begin{aligned}
& \tilde{q}_{n+1}=q_{n}+\Delta t \dot{q}_{n}+\frac{\Delta t^{2}}{2}(1-2 \theta) \ddot{q}_{n} \\
& \tilde{q}_{n+1}=\dot{q}_{n}+\Delta t^{2}(1-\eta) \ddot{q}_{n}
\end{aligned}
$$

wher

$$
\eta=0.5 \quad \text { and } \quad \theta=0.25
$$

Factorize $[\mathrm{G}]$ into $\left[\mathrm{G}_{a}\right]$ and $\left[\mathrm{G}_{\mathrm{n}}\right]$ substitution of equation (37-40) into equation (36) which corresponds to the conventional Lagrange multiplier method leads to

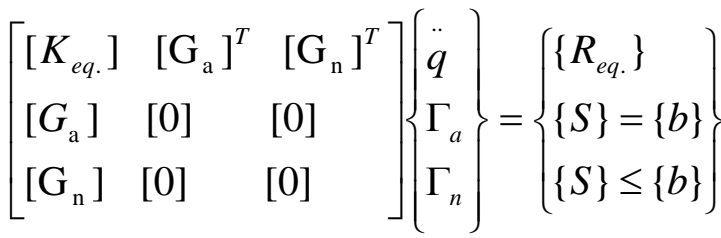

$$
\begin{aligned}
& {\left[K_{\text {eq. }}\right]=[\mathrm{M}]+\eta \Delta \mathrm{t}[\mathrm{C}]+\theta \Delta t^{2}[K]} \\
& \left.\left.\left\{R_{\text {eq. }}\right\}=\{\mathrm{R}\}-[C]\{\dot{\dot{q}}\}-[K]\right\} \tilde{q}\right\}
\end{aligned}
$$

Where;

[Keq.] is the equivalent matrix

[Req.] is the equivalent load vector

$[\mathrm{M}]$ is the mass matrix,

$[\mathrm{C}]$ is the damping matrix,

$[\mathrm{K}]$ is the stiffness matrix,

$\left[\mathrm{G}_{\mathrm{a}}\right]$ is the active constraint matrix,

$\left[\mathrm{G}_{n}\right]$ is the inactive constraint matrix,

$\Gamma_{\mathrm{a}}$ is the Lagrange multiplier corresponding to active subset of constraints have nonzero positive value,

$\Gamma_{\mathrm{n}} \quad$ is the Lagrange multiplier corresponding to inactive subset of constraints should be zero,

$\{b\}$ is the gap vector,

$\{S\}_{a}$ is the correspond to the active constraints, would be equal to corresponding $\{b\}$,

$\{S\}_{n} \quad$ is the correspond to the inactive constraints would be corresponding zero multiplier.

\section{IMPLEMENTATION SCHEME}

The step-by-step implementation scheme is to be performed over the time interval $[0, T]$ as follows:

1- Initialization, set $\mathrm{n}=0$ and define all the initial data. 
2- Form all needed matrices $\{[\mathrm{M}],[\mathrm{C}]$, and $[\mathrm{K}]\}$.

3- Form [Keq.] according to equation (42).

4- Start time step loop.

5- Compute $\tilde{q}_{n+1}$ and $\dot{q}_{n+1}$ according to equations (39) and (40).

6- Compute [Req.] according to $\left.\left.\left.\left\{R_{\text {eq. }}\right\}=\{\mathrm{R}\}-[C]\right\}\{\tilde{\dot{q}}\}-[K]\right\} \tilde{q}\right\}$

7- Solve equation (41) for $\ddot{q}_{n+1}$ and $\Gamma_{a}$.

8- Compute $q_{n+1}$ and $\dot{q}_{n+1}$ according to equations (37) and (38).

9- Check all candidate contact points and detect the contact event and its contact time $\mathrm{T}_{\mathrm{C}}$.

10-Compute the reactions at the contact points and check which is the most probable to release and detect its release time $T_{r}$.

11-Compare $T_{C}$ and $T_{r}$ and chose the minimum to be the state event.

12-Modify [Keq.] according the state event if existed.

13-Add time step

14-If the admissible time is exhausted, STOP.

15-If NOT, Repeat step 4.

\section{NUMERICAL EXAMPLE}

Consider an elastic beam $\boldsymbol{a b}$ of length $I=10 \mathrm{~cm}$, uniform cross-section area with diameter equals one sixth the length, and second moment of area I made of an isotropic homogeneous material has elasticity modulus $\mathbf{E}=210 \times 10^{9} \mathrm{~Pa}$, and mass density $\rho=7.5 \times 10^{3} \mathrm{~kg} / \mathrm{m}^{3}$ impinging a rigid wall with constant velocity $\boldsymbol{v}$ in a plane perpendicular to the wall and the longitudinal axis of the beam inclined at an angle $\varphi$ with the wall at the instant of the first contact as shown in Fig. 4. At the instant $t=0$, the end $\boldsymbol{b}$ of the beam collides with the rigid wall, and the subsequent motion after impact is to be determined from general principles of mechanics subject to certain additional assumptions to be stated.

It is assumed that the duration of the rebound process is not instantaneous but nevertheless is sufficiently small for the beam to remain approximately the same length $I$ as before the impact and inclined at an angle $\alpha$ to the wall. Simultaneously, compressive behavior of the beam is permitted in accordance with standard approximations of the linear theory of elasticity. Rates of change of the displacements to first and higher orders are therefore not necessarily zero during the rebound. Of course, after impact, conversion of the rotational inertia causes the beam immediately to commence bending. The components of the uniform velocity prior to impact are given by $\left(v_{\mathrm{x}} ; v_{\mathrm{y}}\right)$, while, during the period subsequent to contact, the displacement components of any point on the beam are $(x(t) ; y(t))$ and the anti-clockwise rotational displacement is denoted by $\varphi(t)$. 
The proposed model is implemented according to the implementation scheme to solve this example and computational results are obtained. The simulation used 40 transverse and 40 lateral modes of elastic vibration.

Figures showing the contact forces generated over the impact period for friction and frictionless oblique impact taking into consideration the effect of oblique angle $\varphi$. Comparing the contact period with [23] shows good agreement. Figure (5) presents the normal contact forces generated

over the contact period for different incident angels $(45,60,70,80$, and 90$)$ when the coefficient of friction $\mu=1$. Note that normal contact force had built up to a constant value to the last fourth of the contact period then started to fluctuate due to the effect of elastic modes of vibration. Also, the normal contact force decreased as the incident angle $\varphi$ decreased (direct proportionality). Figure (6) shows friction force generated over the contact period. The same behavior is detected but the friction force increased in value as the incident angle decreased (inverse proportionality). The normal contact forces generated when the coefficient of friction $\mu=0$ are presented in Fig. (7). It is depicted that the contact period increased as the incident angle $\varphi$ increased (direct proportionality).

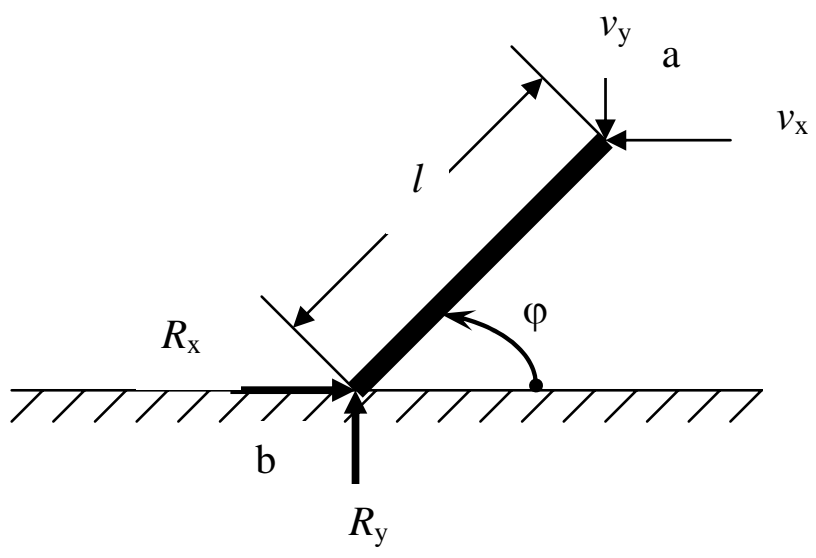

Fig.4.. An Elastic Beam Imbact A Rigid Wall 


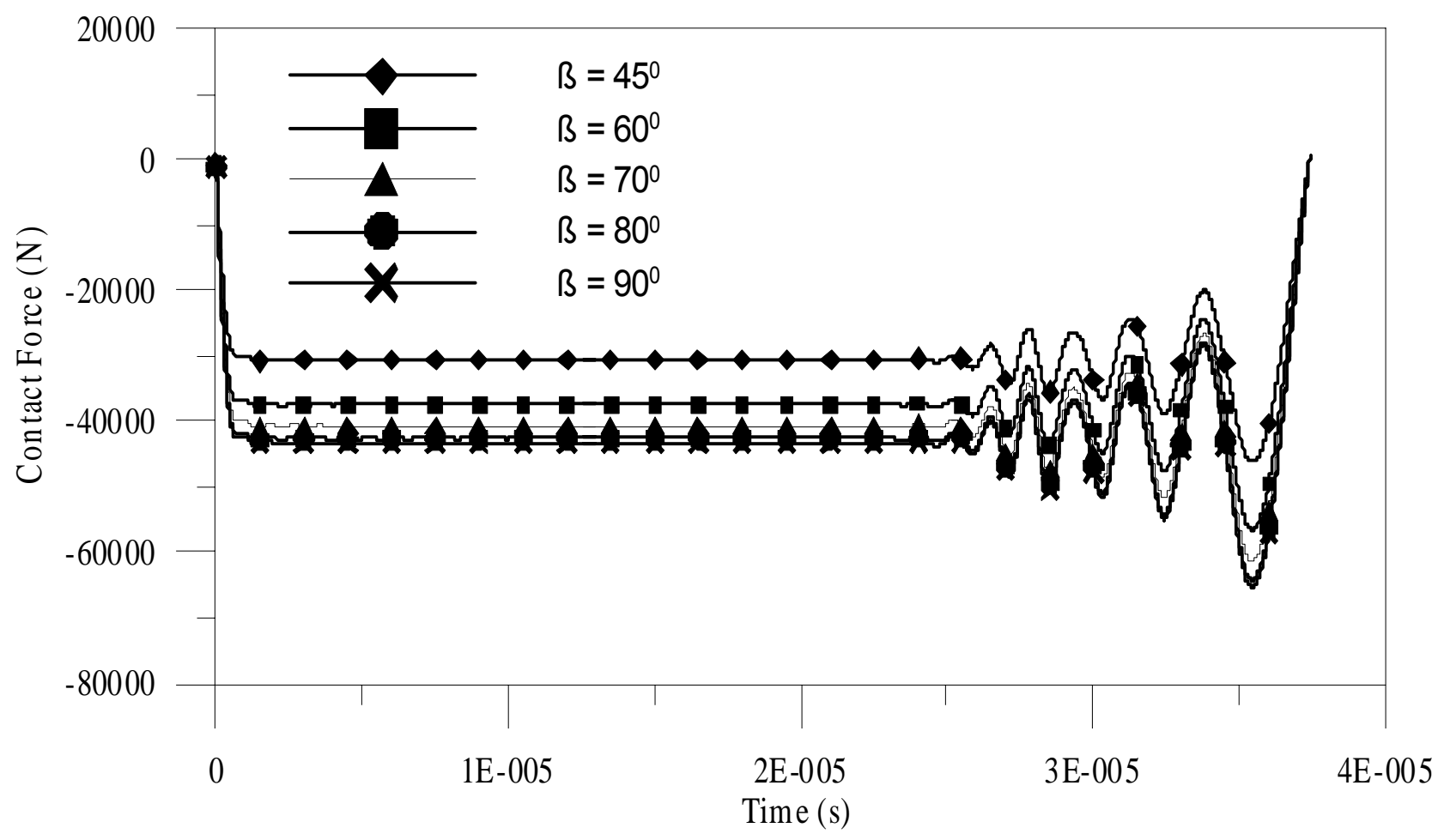

Fig.5. The oblique impact contact force induced by rod at different incident angles $\beta(\mu=1.0)$

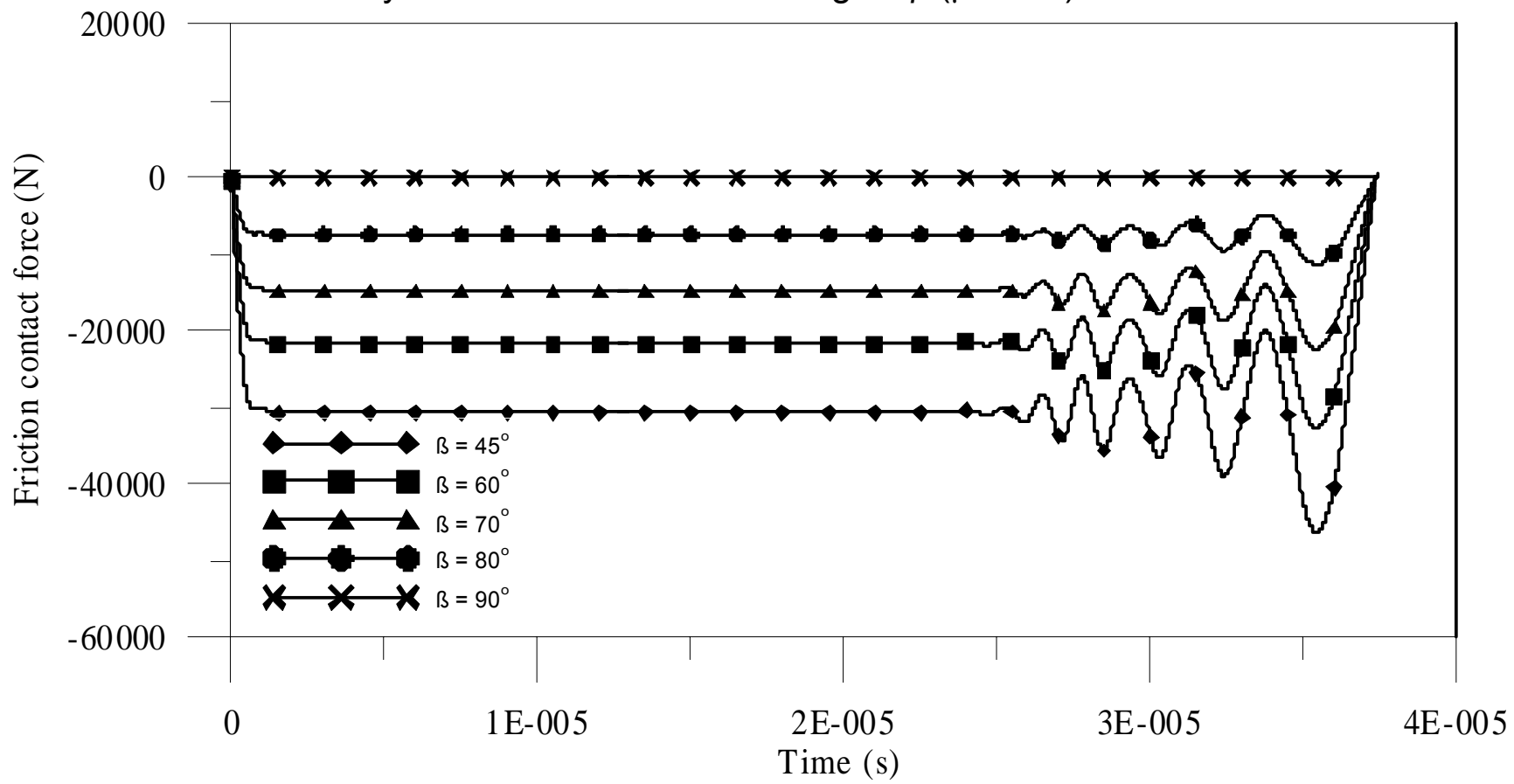

Fig.6. The oblique impact friction force induced by rod at different incident angles $\beta(\mu=1.0)$ 


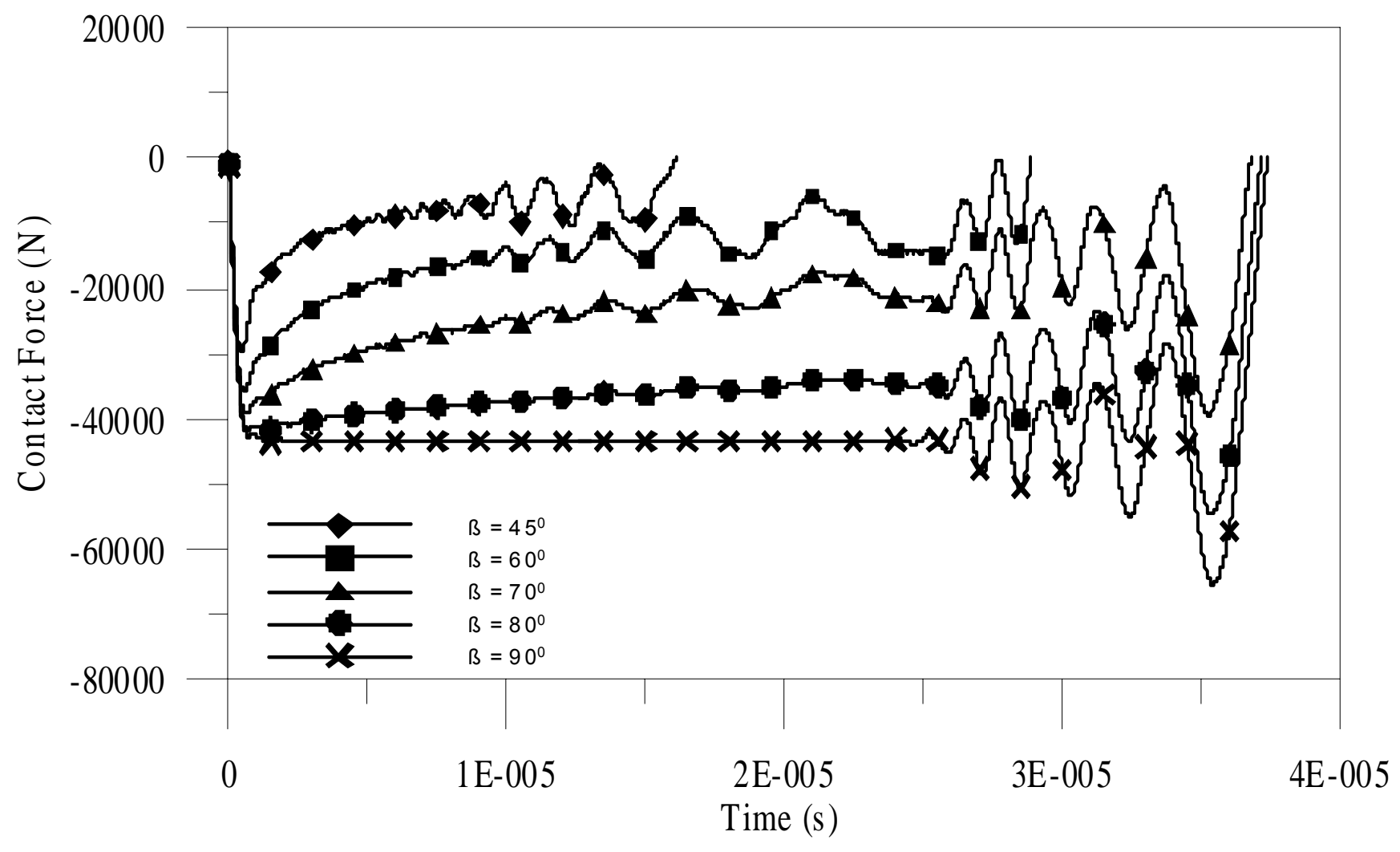

Fig.7. The oblique impact contact force induced by rod at different incident angles $\beta(\mu=0.0)$

Effects of the considered elastic modes of vibration taken into consideration are shown in Fig. (8). It is depicted that the contact force had built up gradually when the beam is presented by one element, classical theory, monotonically compression and monotonically decompression interpretation of Poisson's law of impact. Wherever, as the number of elements increased the constant load period increased. It is also depicted that as the number of elements tends to infinity the normal contact force can approximately represented by a rectangular pulse load with effective time duration $\tau=\pi /$ $\lambda_{n}(\mathrm{~s})$. where $\lambda_{\mathrm{n}}$ is the natural frequency of the system. 


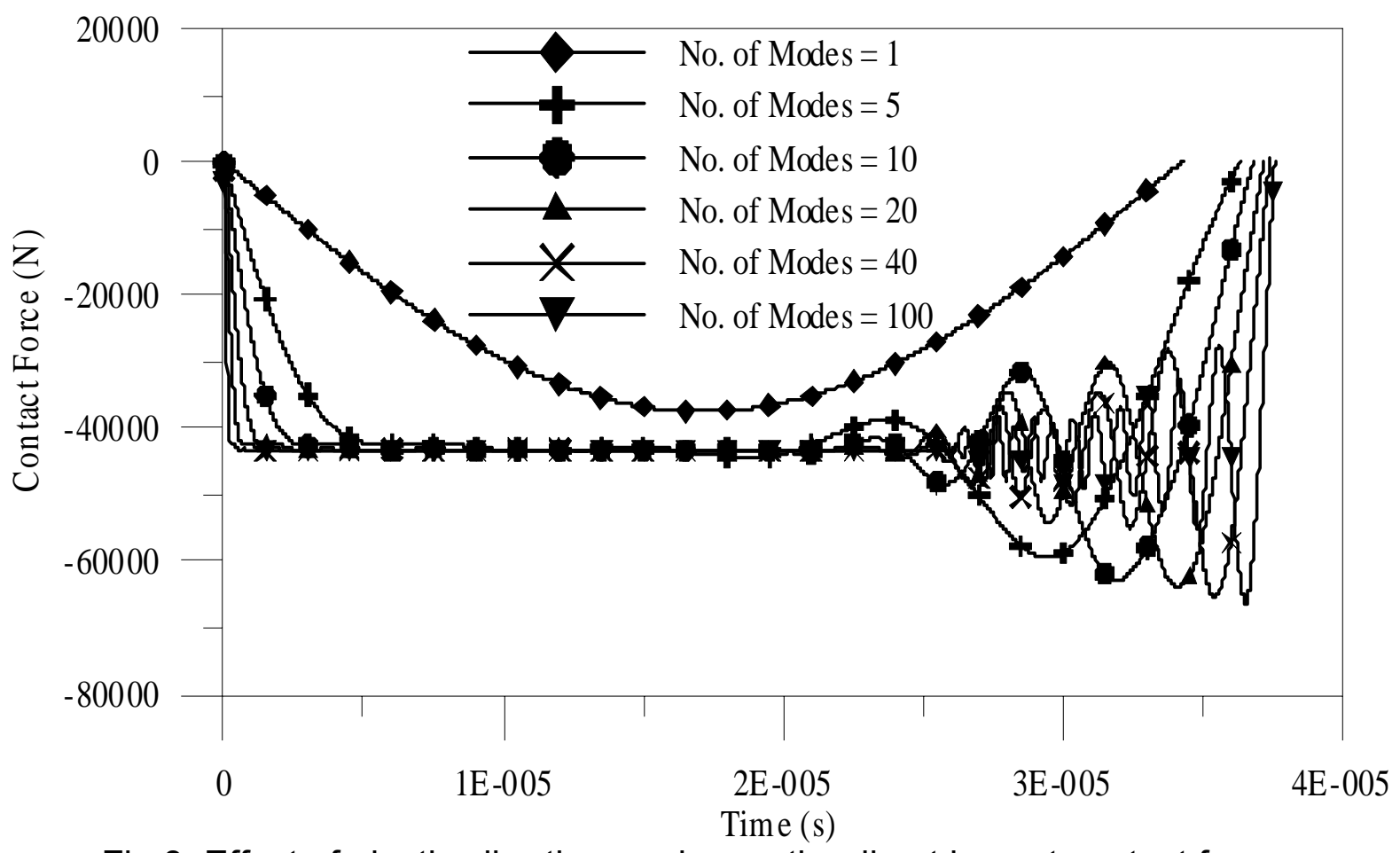

Fig.8. Effect of elastic vibration modes on the direct impact contact forces

\section{CONCLUSION}

An impact model based on Lagrange multiplier and finite element approach has been described and applied to an example involving direct and oblique impact of rod with a rigid obstacle. This example problem studies the effect of oblique angle on impulse force generated within the impact period as well as the neighborhood area of the instantaneous impact model smooth or rough. Moreover, the effect of elastic modes of vibration on the impulsive force generated within the contact period has been discussed. Results show that friction playing a strong role in time contact period as will as in the response of the contact impulsive force. Also, the number of elastic vibration modes taken into consideration had strongly affected the impulse contact force response. 


\section{REFERENCES}

[1] M. Cocu \& J. M. Ricaud, "Existence results for class of implicit evolution inequalities and applications to dynamic unilateral contact problems with friction", C.R. Acad. Sci. Paris Vol. I 329, 839-844, 1999.

[2] J. T. Oden \& E.B. Pires, "Numerical analysis of certain contact problems in elasticity with non-classical friction law", Computer and structure, Vol. 16, 481485, 1983.

[3] J. T. Oden \& E.B. Pires, " Algorithm and numerical results for finite element approximation of contact problems with non-classical friction law", Computer and structure, Vol. 19, 137-147, 1984.

[4] J. T. Oden \& J. A. C. Martins, "Models and computation methods for dynamic friction phenomena" Computer Meth. Appl. Mech. Engng. 52, 527-634, 1985.

[5] J. A. C. Martins \& J. T. Oden, "Existence and uniqueness results for dynamic contact problems with nonlinear normal and friction interface laws", Nonlinear Analysis 11, 407-428, 1987.

[6] N. Kikuchi \& J. T. Oden, "Dynamic contact problem in elasticity: a study of variational inequalities and finite element methods", SIAM Studies in Applied Mathematics, no. 8. Philadelphia, PA: SIAM, 1988.

[7] D. E. Stewart, "Convergence of a time stepping scheme for rigid body dynamics and resolution of Painleves problems", Arch. Rotation Mech. Analysis 145, 215260, 1998.

[8] D. E. Stewart, "Formulating measure differential inclusion in infinite dimensions", J. Set-Valued Analysis 8, 273-293, 2000.

[9] D. E. Stewart, "Rigid body dynamics with friction and impact", SIAM Rev. 42, 339, 2000.

[10] D. E. Stewart, "Finite dimension Contact mechanics" Phil. Trans. R. Soc. Lond. A, 359, 2467-2482, 2001.

[11] J. M. Solberg, P. Papadopouslos, "Impact of an elastic pseudo-rigid body on a rigid foundation", Int. J. Engng Sci. 38, 589-603, 2000.

[12] M. Schatzman, "Penalty method for impact in generalized coordinates", Phil. Trans. R. Soc. Lond. A, 359, 2429-2446, 2001.

[13] C. Glocker, "On friction less impact models in rigid-body systems", Phil. Trans. R. Soc. Lond. A, 359, 2385-2404, 2001.

[14] L. Paoli, "Time discretization of vibro-impact", Phil. Trans. R. Soc. Lond. A, 359, 2405-2428, 2001.

[15] F. F. Mahmoud, M. M. Hassan, and N. J. Salamon, "Dynamic contact of deformable bodies", Computer \& Structure, Vol. 36, No. 1, 169-181, 1990.

[16] T. J. Hughes, R. L. Taylor, J. L. Sackman, A. Curnier \& w. Kanoknukulchai, "A finite element method for a class of contact impact problems", Comut. Meth. Appl. Mech. Engng., 8, 249-276, 1976.

[17] L. A. Hageman \& D. M. Yang, "Applied iterative methods", Academic Press, 1981.

[18] K. J. Bathe \& E. L. Wilson, "Numerical methods in finite element analysis", Prentice-Hall, Inc., Englewood Cliffs, New Jersey, 1976.

[19] O. C. Zienkiewicz \& R. L. Taylor, " The finite element method", Fourth edition, McGraw-Hill, UK, 1989. 
[20] T. J. R. Hughes \& W. K. Lui., "Implicit-Explicit finite element in transient analysis stability theory", J. Appl. Mech., 45, 371-374, 1978.

[21] T. J. R. Hughes \& W. K. Lui., "Implicit-Explicit finite element in transient analysis: Implementation and numerical examples", J. Appl. Mech., 45, 375-378, 1978.

[22] T. J. R. Hughes, K. S. Pister \& R. L. Taylor, "Implicit-Explicit finite element in nonlinear transient analysis", Comp. Meth. Appl. Mech. \& Engng., 17/18, 159-182, 1979.

[23] R. J. Knops and P. Villaggio, "An elementary theory of the oblique impact of rods", Rend. Math. Acc. Lincei s. 9,v.12, 49-56, 2001. 